\title{
Andreas Pietsch, Barbara Stollberg-Rilinger (dir.), Konfessionelle Ambiguität. Uneindeutigkeit und Verstellung als religiöse Praxis in der Frühen Neuzeit
}

Gütersloh : Gütersloher Verlagshaus (Schriften des Vereins für Reformationsgeschichte, 214), 2013, 379 p., 39,99€

Juliette Guilbaud

\section{CpenEdition}

\section{Journals}

Édition électronique

URL : http://journals.openedition.org/ifha/8106

DOI : $10.4000 /$ ifha. 8106

ISSN : 2198-8943

Éditeur

IFRA - Institut franco-allemand (sciences historiques et sociales)

Référence électronique

Juliette Guilbaud, « Andreas Pietsch, Barbara Stollberg-Rilinger (dir.), Konfessionelle Ambiguität Uneindeutigkeit und Verstellung als religiöse Praxis in der Frühen Neuzeit », Revue de l'IFHA [En ligne], Date de recension, mis en ligne le 14 avril 2015, consulté le 22 septembre 2020. URL : http:// journals.openedition.org/ifha/8106 ; DOI : https://doi.org/10.4000/ifha.8106

Ce document a été généré automatiquement le 22 septembre 2020.

(CIFHA 


\section{Andreas Pietsch, Barbara Stollberg- Rilinger (dir.), Konfessionelle Ambiguität. Uneindeutigkeit und Verstellung als religiöse Praxis in der Frühen Neuzeit}

Gütersloh : Gütersloher Verlagshaus (Schriften des Vereins für Reformationsgeschichte, 214), 2013, 379 p., 39,99€

Juliette Guilbaud

1 Le paysage académique allemand, à l'instar de celui de la France, est depuis plusieurs années bouleversé par la mise en place de superstructures et autres pôles d'excellence. C'est dans le cadre de l'un de ces clusters d'excellence que s'est développé le projet piloté par B.Stollberg-Rilinger et A. Pietsch, intitulé «Politique et dissimulation religieuse. Ambiguïté confessionnelle aux XVI ${ }^{\mathrm{e}}$ et $\mathrm{XVII}^{\mathrm{e}}$ siècles ». Ce projet a fait l'objet en 2010 d'un colloque à Münster qui rassemblait historiens, littéraires, linguistes, théologiens, historiens de l'art et philosophes, et dont le présent volume est pour partie la publication. Pour partie, car aux contributions présentées oralement ont été ajoutés une copieuse introduction de la codirectrice de l'ouvrage ainsi que cinq papiers qui permettent d'élargir le cadre de l'analyse au-delà de la seule Europe occidentale. L'introduction est un modèle du genre, qui combine à la fois un rappel des questions qui ont sous-tendu le sujet du colloque, une synthèse des seize contributions rassemblées et, pour finir, une mise en perspective de l'ensemble, laquelle aurait aussi bien pu faire figure de conclusion.

2 Au départ du colloque figurait le constat d'une conjoncture favorable à la dissimulation et à la simulation à l'époque moderne, en particulier durant la période de la confessionnalisation. De quoi était-elle la traduction? Mais d'abord, qu'est-ce que l'ambiguïté religieuse ou confessionnelle? En quoi peut-elle se révéler problématique, en particulier aux $\mathrm{XVI}^{\mathrm{e}}-\mathrm{XVII}{ }^{\mathrm{e}}$ siècles ? Dans une historiographie où le paradigme de la 
confessionnalisation imprègne fortement les travaux d'histoire et d'anthropologie religieuses - et contrairement à la France ou à l'Angleterre -, ces questions ont encore fait l'objet d'assez peu d'études (voir par exemple le volume dirigé par G. Mühlpfordt et U. Weiß, Kryptoradikalität in der Frühen Neuzeit, Stuttgart, 2009 ; http://ifha.revues.org/ 6657). Les questions soulevées en introduction montrent la nécessité de s'interroger sur la pertinence des catégories d'analyse et, pour le cas précis de la différenciation religieuse, sur le point de départ de toute définition : indifférence/tolérance, religion intérieure/extérieure, dimension rituelle/théorique, discours/pratiques.

3 L'agencement des contributions dans le volume permet de dégager trois grands axes de réflexion. Le premier volet propose une analyse à la fois sémantique et théorique des différents concepts utilisés: la confession, comme profession de foi du croyant et confession de foi d'une communauté (Ph. Büttgen, p. 27-38) ; l'indifférence, qui n'est ni tolérance, ni méconnaissance, ni blasphème (K. von Greyerz, p. 39-61) ; le nicodémisme, comme attitude commune en dehors de l'orthodoxie (J.-P.Cavaillé, p. 62-74); les pratiques cryptoreligieuses dans l'Empire ottoman comme révélateurs d'une " métadoxie », définie comme cet état de pluralité et de porosité religieuses antérieur à l'élaboration de dogmes et dégagé, par conséquent, des notions d'ortho- ou d'hétérodoxie (M. Reinkowski, p. 75-98); la dissimulatio et la simulatio à l'exemple des Essais de Montaigne (K. Westerwelle, p. 118-141). J. Macha défend pour sa part la thèse que certains marqueurs linguistiques, qu'il décrit comme confessionnels (mais en quoi le sont-ils précisément?), dépassent le cadre de la doctrine et du culte pour se retrouver dans la vie quotidienne (p. 99-117).

4 Le deuxième volet balaie à travers six études de cas le spectre de l'ambiguïté religieuse dans les milieux privilégiés et/ou lettrés, ce que M Pohlig a appelé la «dévotion savante» («gelehrter Frömmigkeitsstil »). La labilité des frontières confessionnelles est manifeste et la question est autant religieuse que politique dans le conflit autour de Frédéric III de Palatinat, lors de la diète de 1566 (M. Pohlig, p. 142-169). Les contributions sur la conversion du duc de Lesdiguières (J.-F. Mißfelder, p. 170-182) ou celle du comte de Schaumburg-Lippe (L. Baibl, p. 183-209), dans le courant des nouvelles recherches sur les conversions de l'époque moderne, s'interrogent pour leur part plus sur les stratégies de communication que sur les motifs et la sincérité (ou non) de ces démarches individuelles. Paradoxalement, il semble que les milieux savants ou académiques aient pu faciliter le développement de positions religieuses relativement floues d'un point de vue dogmatique, comme le montrent les exemples de Daniel Casper von Lohenstein (S. Neumeister, p. 210-220), des sociniens d'Altdorf (F. Vollhardt, p. 221-237) ou de Juste Lipse et Hendrik Niclaes, le fondateur de la familia caritatis en 1540 (A. Pietsch, p. 238-266).

5 Le troisième et dernier volet s'extrait du strict contexte de la confessionnalisation pour s'intéresser à la question de l'ambiguïté religieuse en matière de dogme et de sacrements: chez les remonstrants des Provinces-Unies au début $d u$ XvII $^{\mathrm{e}}$ siècle (J. Müller, p. 267-284) ; dans l'Angleterre du Xvi e siècle (A. Walsham, p. 285-313); dans le cadre des missions catholiques auprès des chrétiens arméniens de l'empire safavide en Perse aux XVII ${ }^{\mathrm{e}}$-XVIII ${ }^{\mathrm{e}}$ siècles (Ch. Windler, p. 314-345); et chez les cryptoprotestants autrichiens, particulièrement persécutés à partir des années 1750 jusqu'à la patente de tolérance de Joseph II (M. Scheutz, p. 346-372).

6 L'ensemble de ces contributions appelle une menue critique : on peut regretter que la plupart des études de cas présentées ici concerne des milieux relativement privilégiés 
ou des cas très spécifiques. Il serait intéressant de pouvoir se pencher sur l'ambiguïté confessionnelle ou religieuse en milieu rural où vit la plus grande part des populations. Plusieurs remarques pour terminer. La première est, sans conteste, que l'ambiguïté en matière religieuse n'est pas chose rare à l'époque moderne et, comme bien des phénomènes, c'est à travers les conflits qu'elle est le mieux mise en lumière car ceux-ci sont producteurs de sources. La question de l'orthodoxie/hétérodoxie semble d'autant plus marquante durant la période de la confessionnalisation que celle-ci se caractérise notamment par la définition juridique de ce que sont les confessions. Les limites entre orthodoxie - réputée stable et intangible de son propre point de vue - et hétérodoxies sont loin d'être définitivement fixées. Les frontières confessionnelles, dans les pratiques comme dans l'espace, comme l'a par ailleurs bien montré Ch. Duhamelle ( $L a$ Frontière au village..., Paris, 2010), font l'objet d'un rejeu permanent. Enfin et surtout, l'historien doit se garder d'adopter par commodité le point de vue des «vainqueurs». Pour la justesse de ses définitions, la diversité des points de vue adoptés et le salutaire pas de côté qu'il propose par rapport au contexte de l'Europe occidentale moderne, ce volume est une lecture hautement recommandable.

INDEX

Index chronologique : Période moderne

Thèmes : Histoire religieuse, Histoire sociale

\section{AUTEUR}

JULIETTE GUILBAUD

CNRS, centre Georg-Simmel (UMR 8131) 